\title{
Modelado y control de un sistema de levitación magnética basado en un cojinete magnético activo
}

\section{Modeling and control of a magnetic levitation system based on active magnetic bearing}

\author{
Marlon Cruz Pegueros ${ }^{1}$, O. Octavio Gutiérrez Frías ${ }^{1}$, Norma B. Lozada Castillo ${ }^{2}$ y Alberto \\ Luviano Juárez ${ }^{1}$
}

Palabras Clave: Cojinete magnético activo; control lineal; fuerza electromagnética; plataforma de pruebas

\section{Recepción: 03-04-17 / Aceptación: 24-08-17}

\section{Resumen}

En este trabajo se presenta el diseño, modelado e implementación de un sistema de levitación magnética de un grado de libertad, que consiste en una viga actuada por un cojinete magnético activo en configuración pendular. Se obtiene su representación en espacio de estado y, tras una linealización tangente, su función de transferencia correspondiente. Se describen las características del dispositivo experimental, su instrumentación electrónica y mecanismo, particularmente el procedimiento de diseño del actuador electromagnético empleado, así como su caracterización para determinar la constante de proporcionalidad de la fuerza electromagnética. Finalmente, se muestra la simulación numérica e implementación experimental de esquemas clásicos de control para estabilizar en el punto de equilibrio del sistema

\begin{abstract}
In this paper, we present the design, modeling as well as the implementation of a magnetic levitation system, consisting on a single-degree of freedom pendular beam, which is driven by means of an active magnetic bearing. The analysis includes the state space representation and, after a tangent linearization process, its transfer function is obtained. In addition, the characteristics of the device such as the electronic instrumentation and mechanical design are described. In particular, it is shown the design procedure of the electromagnetic actuator used, as well as its characterization to determine the proportional constant of the electromagnetic force with respect to a current input. Finally, some numerical simulations and experimental results of the implementation of some classic controllers in stabilization task are shown.
\end{abstract}


Cruz Pegueros, Marlon et al.

Keywords: Active magnetic bearing; linear control; electromagnetic force; test bed. 


\section{Introducción}

Los sistemas de levitación magnética en los últimos años se han considerado como un banco de pruebas para diversas estrategias de control (Lei, 2008), (Golob, 2003), (Qin, 2014), (Bächle, 2013) y (Beltran, 2015). Además, se han popularizado por las diversas aplicaciones que tienen, tales como: cojinetes magnéticos (Chen, 2010) y (Du, 2010); sistemas para trenes de levitación magnética (Hasirci, 2011); aislamiento de vibraciones (Tsuda, 2009); microrobots magnéticos (Kummer, 2010); máquinas eléctricas (Arrendondo, 2008); sistema de transportación magnética (Wai, 2011); sistemas de posicionamiento nanométrico (Kim, 2007), entre muchas otras (Peijnenburg, 2006), (Kimman, 2010), (Lee, 2006). El sistema de levitación propuesto en este trabajo consiste en una viga con libertad para rotar, la cual es estabilizada mediante un electroimán colocado en uno de los extremos. El objetivo principal de control en el sistema, es mantener la viga en una posición deseada. En lo que se refiere al control de este sistema existen en la literatura algunos trabajos que abordan el tema. Lindlau et al. (Lindlau, 2002) presentan un controlador mediante linealización por realimentación y, observando su funcionamiento mediante la medición de salida ante entradas lineales, se muestran resultados experimentales del comportamiento del sistema. En Mahindrakar (2008) se presenta un esquema de control para estabilizar el sistema, tomando en cuenta que tiene entrada y estados restringidos, proponiendo un controlador basado en Lyapunov, y realizando la comparación con una ley de control lineal. Otro trabajo que aborda el problema, es el propuesto por $\mathrm{Hu}$ et al. (Hu, 2005) en el que se desarrolla un enfoque de diseño sistemático de control con entrada y estados restringidos, obteniendo una región de atracción mayor, comparada con las obtenidas en técnicas lineales; los resultados fueron verificados de manera experimental. En Rodríguez (2000) se usa el método de pasividad para resolver el problema de estabilización del sistema de levitación magnética. Otro trabajo interesante es la propuesta de Lee et al. (Lee, 2003), que soluciona el problema de control de este sistema mediante control por modos deslizantes utilizando dos actuadores magnéticos.

En este artículo, se presenta el modelado del sistema de levitación magnética de un grado de libertad, así como la descripción del dispositivo de pruebas con los elementos electrónicos y mecánicos para su implementación. Además, se menciona el procedimiento de diseño del actuador electromagnético. Por otra parte, se presenta la simulación e implementación de un controlador tipo Proporcional Integral Derivativo (PID) para observar su funcionamiento, así como uno de tipo Proporcional Integral (PI). La principal contribución del trabajo consiste en el 
sistema que puede funcionar como banco de pruebas para la implementación de diferentes estrategias de control. El trabajo se organiza de la siguiente manera: la sección 2 describe el modelo dinámico del sistema de levitación magnética. En la sección 3 se mencionan las características de la plataforma experimental, además de presentar un procedimiento de diseño del actuador electromagnético. La sección 4 presenta la estrategia de control de tipo PID y algunas simulaciones numéricas. La sección 5 incluye los resultados experimentales implementando la estrategia de control en la plataforma experimental. Finalmente, la sección 6 está dedicada a las conclusiones.

\section{Modelado del Sistema}

El Sistema de levitación magnética, mostrado en la Figura 1, es un sistema electromecánico que consiste en una viga en desequilibrio, cuyo centro de gravedad se encuentra a una distancia $r$. Así mismo, el dispositivo cuenta con un imán permanente de neodimio a una distancia $l$ con respecto al pivote de la viga, además de un electroimán que tiene la función de actuador del sistema. El modelo no lineal es una variante de los sistemas propuestos en Lindlau (2002), Mahindrakar (2007), y sus ecuaciones diferenciales pueden ser obtenidas de las ecuaciones de Euler-Lagrange y las Leyes de Kirchoff, definidas de la siguiente manera:

$$
\begin{gathered}
\frac{d^{2} \theta}{d t}=\frac{1}{J} C_{e} \frac{i^{2}}{(l \sin \theta)^{2}}-\frac{m g r \cos \theta}{J} \\
\frac{d i}{d t}=-\frac{R}{L} i+\frac{1}{L} u
\end{gathered}
$$

Donde:

- $\theta$ : ángulo de la viga con la horizontal;

- $\quad i$ : corriente eléctrica que circula por la bobina del electroimán;

- $m$ y J: parámetros correspondientes a la masa y momento de inercia de la viga respectivamente;

- $\quad l$ : distancia entre el pivote y el centro del imán permanente;

- $\quad r$ : distancia radial del centro de masa de la viga al centro de rotación;

- $g:$ constante de gravedad;

- $\quad C_{e}$ : constante de proporcionalidad de la fuerza electromagnética; 
- $R, L:$ resistencia y la inductancia de la bobina del subsistema electromagnético, respectivamente;

- $\quad u$ : entrada de voltaje aplicada al sistema electromagnético.

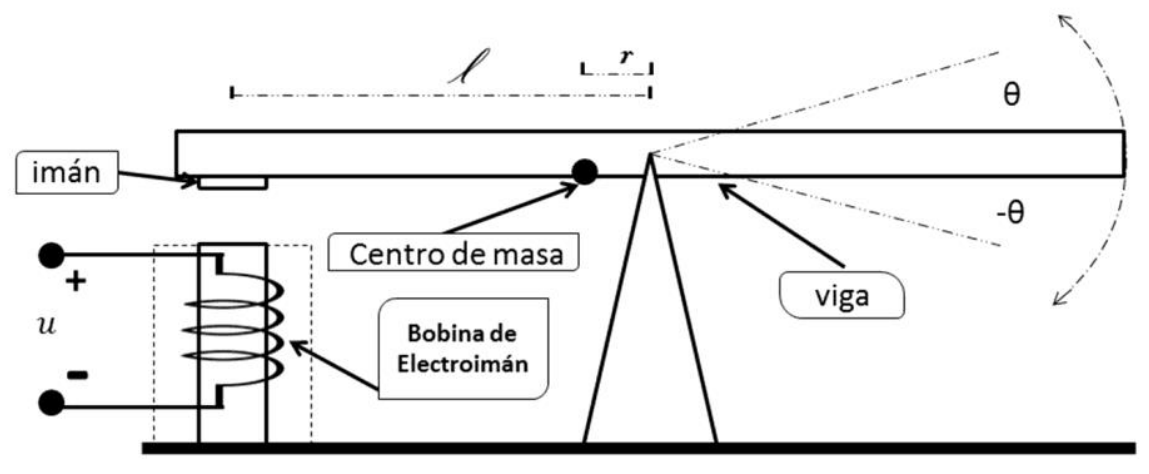

Figura 1. Sistema de levitación magnética.

Se definen las siguientes variables de estado para el sistema (1):

$$
\begin{aligned}
& x_{1}=\theta \\
& x_{2}=\dot{\theta} \\
& x_{3}=i
\end{aligned}
$$

$X=\left(x_{1}, x_{2}, x_{3}\right)^{T}$, el vector de estado correspondiente. El sistema no lineal en variables de estado es expresado de la siguiente forma:

$$
\begin{gathered}
\frac{d x_{1}}{d t}=x_{2} \\
\frac{d x_{2}}{d t}=\frac{c_{e}}{J}\left(\frac{x_{3}}{l \sin x_{1}}\right)^{2}-\frac{m g r \cos x_{1}}{J} \\
\frac{d x_{3}}{d t}=-\frac{R x_{3}}{L}+\frac{u}{L}
\end{gathered}
$$

Para obtener el punto de equilibrio se considera $\frac{d x_{1}}{d t}=\frac{d x_{2}}{d t}=\frac{d x_{3}}{d t}=0$, luego se tiene:

$$
\begin{gathered}
\frac{d x_{1}}{d t}=0 \text {, implica que } x_{2}=0 \mathrm{y} x_{1}=\bar{x} \operatorname{con} \bar{x} \text { un valor real } \\
\frac{d x_{2}}{d t}=0, \quad \frac{d x_{2}}{d t}=\frac{C_{e}}{J}\left(\frac{x_{3}}{l \sin x_{1}}\right)^{2}-\frac{m g r \cos x_{1}}{J}=0 \Rightarrow\left(\frac{x_{3}}{l \sin x_{1}}\right)^{2}=\frac{m g r \cos x_{1}}{C_{e}}
\end{gathered}
$$




$$
x_{3}=\sqrt{\frac{\left(m g r \cos x_{1}\right)}{C_{e}}} l \sin x_{1} \Rightarrow x_{3}=\sqrt{\frac{\left(m g r \cos x_{1}\right)\left(l \sin x_{1}\right)^{2}}{C_{e}}}
$$

Considerando la naturaleza de $x_{1}$ se reescribe

$$
\begin{gathered}
x_{3}=\sqrt{\frac{(m g r \cos \bar{x})(l \sin \bar{x})^{2}}{C_{e}}} \\
\frac{d x_{3}}{d t}=0, \\
\frac{d x_{3}}{d t}=-\frac{R x_{3}}{L}+\frac{u}{L}=0 \Rightarrow \frac{u}{L}=\frac{R x_{3}}{L} \Rightarrow \mathrm{u}=R x_{3}
\end{gathered}
$$

Considerando $x_{3}, \mathrm{u}=R \sqrt{\frac{(m g r \cos \bar{x})(l \sin \bar{x})^{2}}{C_{e}}}$.

Finalmente, el punto de equilibrio está dado por:

$$
\bar{X}=\left(\bar{x}_{1}, \bar{x}_{2}, \bar{x}_{3}\right)=\left(\bar{x}, 0, \sqrt{(g m r \cos \bar{x})(l \sin \bar{x})^{2} / C_{e}}\right) .
$$

Se puede calcular la entrada de control $u$ en estado permanente para mantener la viga en la posición deseada $x_{1}^{*}$ de la siguiente manera:

$$
\bar{u}=R \sqrt{\frac{(g m r \cos \bar{x})(l \sin \bar{x})^{2}}{C_{e}}}
$$

Donde se puede deducir que en estado permanente es puramente resistiva y que los valores de la variable de corriente y de la entrada de control se incrementan cuando la viga se aleja de la horizontal. Es importante notar que si $x_{1}=0$ se tiene una singularidad en $\dot{x}_{2}$ para evitarlo, se considera un valor cercano, a saber, $x_{1}=0.001 \mathrm{rad}$.

Formulación del problema: El objetivo primordial es estabilizar el sistema alrededor del punto de equilibrio, a través de una estrategia de control clásica, suponiendo que la viga se encuentra 
inicialmente en la región $x_{1 . s}=\left(-x_{1 \max }, x_{1 \max }\right)$ con $x_{1 \max }$ fijo en los valores de operación. En otras palabras, la intención es llevar la viga a la posición deseada $x_{1}^{*}$.

Para el cálculo de la ley de control estabilizante se utiliza la linealización aproximada del sistema no lineal respecto al punto de equilibrio $\bar{X}$, la cual tiene la siguiente expresión:

$$
\begin{array}{r}
\frac{d X}{d t}=A X+B u \\
Y=C X
\end{array}
$$

Donde:

$$
\begin{gathered}
A=\left[\begin{array}{ccc}
0 & 1 & 0 \\
\frac{1}{J}\left(g m r-2 g m r \cot ^{2} \bar{x}_{1}\right) \sin \bar{x}_{1} & 0 & \frac{2 C_{e} \csc ^{2} \bar{x}_{1} \sqrt{\frac{g l^{2} m r \cos \bar{x}_{1} \sin ^{2} \bar{x}_{1}}{C_{e}}}}{J l^{2}} \\
0 & 0 & -\frac{R}{L}
\end{array}\right] ; \quad B=\left[\begin{array}{l}
0 \\
0 \\
\frac{1}{L}
\end{array}\right] \\
C=\left[\begin{array}{lll}
1 & 0 & 0
\end{array}\right]
\end{gathered}
$$

De la ecuación (4) se puede obtener la función de transferencia del sistema, expresada de la siguiente forma:

$$
G_{p}(s)=\frac{Y(s)}{u(s)}=C(s I-A)^{-1} B=\frac{4 \mathrm{C}_{e} \csc \bar{x}_{1} \sqrt{\frac{g l^{2} m r \cos \bar{x}_{1} \sin ^{2} \bar{x}_{1}}{\mathrm{C}_{e}}}}{l^{2}(R+L s)\left(g m r\left(1+3 \cos 2 \bar{x}_{1}\right)+2 J s^{2} \sin \bar{x}_{1}\right)}
$$

La ecuación anterior es la función de transferencia del sistema (4), donde la entrada es el voltaje aplicado a la bobina -denotado por la variable $u$ - y la salida la posición angular de la viga denotada por la variable Y-.

\section{Plataforma experimental}

En esta sección se describe la plataforma experimental utilizada (Figura 2), mencionando los dispositivos utilizados para la medición de la salida, así como el procedimiento para determinar la constante proporcional de la fuerza electromagnética. 


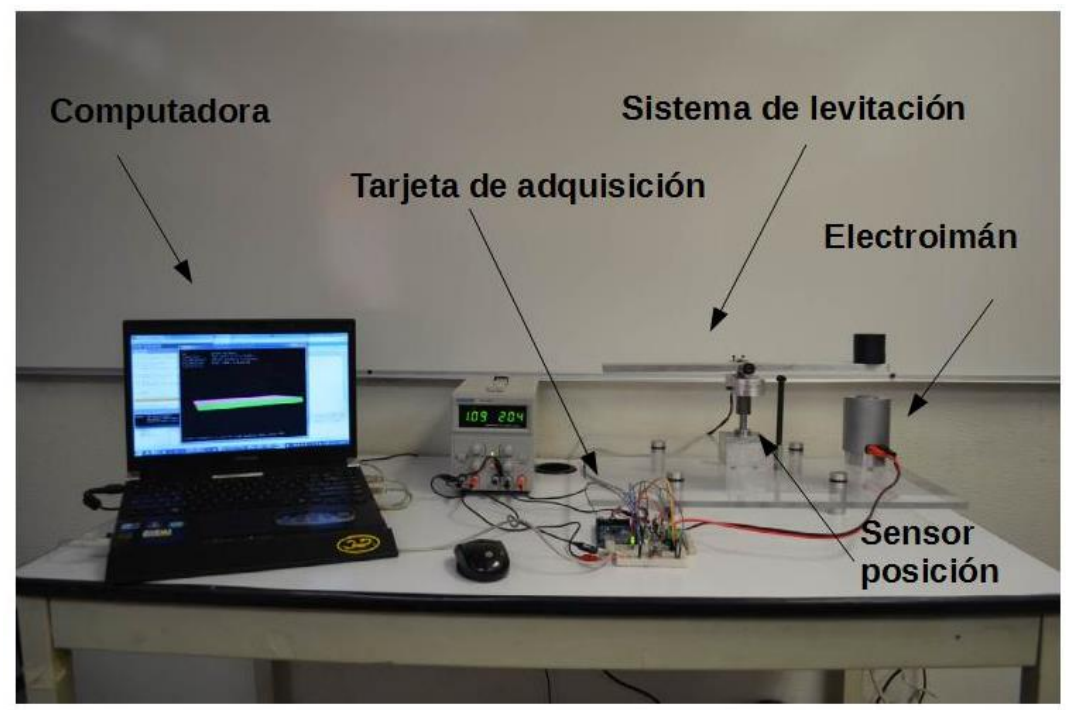

Figura 2. Plataforma experimental del sistema de levitación magnética.

\section{Descripción de la plataforma}

La plataforma consiste en:

- Una viga de aluminio de aleación temple estándar, por sus características de fácil maquinado; cuenta con propiedades paramagnéticas, asegurando que no se magnetice dicha viga con un imán de neodimio colocado en un extremo para lograr la interacción entre campos magnéticos.

- Sensor ACS714 de la compañía Allegro con valores de operación 5V de alimentación y una sensibilidad de $60 \mathrm{mV} / \mathrm{A}$, para la medición de la corriente $\left(x_{3}=i\right)$.

- Sensor IMU 6DOF-I2C que integra un acelerómetro ADXL345 y un giroscopio ITG3200 que permite medir la posición angular de la viga con respecto a la vertical $\left(x_{1}=\theta\right)$.

- Bobina, ver Figura 4.

- Tarjeta Discovery STM32F4 para leer las variables del sistema, calcular la acción de control y enviar datos por puerto serial a una computadora.

Los datos obtenidos son analizados a través del software MATLAB-SIMULINK versión 2013 de 64bits en una computadora con procesador Core i5 de cuarta generación con 6 Gb RAM. Pictóricamente, se describe la plataforma en el siguiente diagrama a bloques, ver Figura 3. 
Modelado y control de un sistema de levitación magnética basado en un cojinete magnético activo

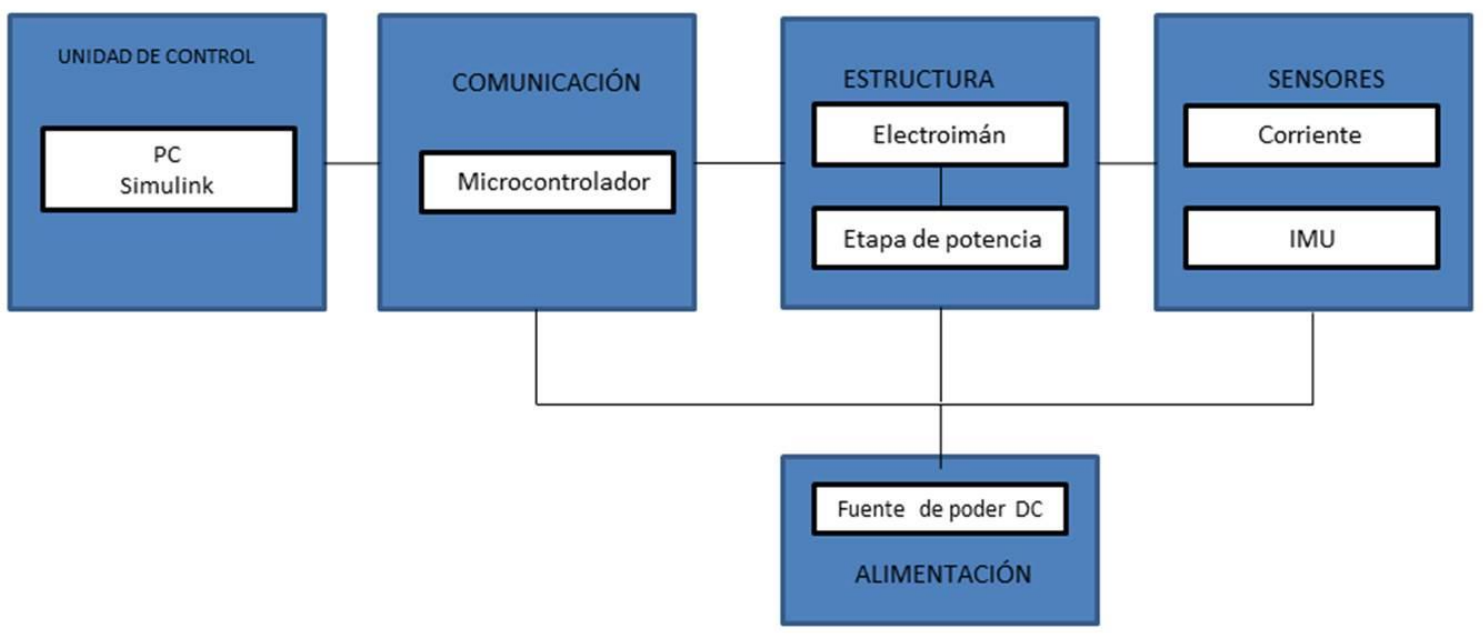

Figura 3. Diagrama a bloques de la plataforma experimental.

\section{Descripción del diseño del electroimán}

En Rajeshkanna (2012) se describe una metodología para el diseño del electroimán, dicha propuesta es considerada en el presente trabajo. A continuación se presentan los cálculos para diseño del actuador empleado en la plataforma.

Como primer aspecto a considerar, se requiere un electroimán con la capacidad de mover una masa de $0.2 \mathrm{~kg}$ a una distancia de $0.05 \mathrm{~m}$. Por tanto, se proponen las siguientes dimensiones para el núcleo y constantes: área de la cara polar $(A)=5.07 \times 10^{-4} \mathrm{~m}^{2}$, longitud del cilindro $(L)=66 \mathrm{~mm}$, diámetro del cilindro $(D)=23 \mathrm{~mm}$, área total del cilindro $\left(A_{t}\right)=56 \times 10^{-4} \mathrm{~m}^{2} \mathrm{y}$ permeabilidad magnética $\left(\mu_{0}\right)=4 \pi \times 10^{-7} \mathrm{Hm}^{-1}$.

Luego, note que al energizar el electroimán se busca una fuerza adecuada para que mantenga un estado de equilibrio para la masa de la viga. Por tanto, se tiene la siguiente restricción:

$$
F_{\text {mag }}=F_{\text {gravedad }}
$$

Por otra parte, debido al peso de la masa de la viga (m) de $0.2 \mathrm{~kg}$, la fuerza es:

$$
F_{\text {gravedad }}=m g=(0.2 \mathrm{Kg})\left(9.81 \mathrm{~m} / \mathrm{s}^{2}\right)=1.962 \mathrm{~N}
$$


Entonces, para mantener o mover la viga en equilibrio, se requiere una fuerza magnética igual o mayor a $1.962 \mathrm{~N}$; de este resultado se partirá para obtener el valor máximo de la corriente que circulará por el electroimán. De las expresiones (6) y (7) se obtiene:

$$
F_{m a g}=\frac{B^{2} A}{2 \mu_{0}}
$$

Y despejando el valor de la densidad de flujo $(B)$, se tiene:

$$
B=0.09862 \frac{w b}{m^{2}}
$$

Por lo anterior, la fuerza magneto-motriz (fmm) definida como:

$$
f m m=\frac{B}{\mu_{0}} L
$$

Con una longitud del entrehierro $(L)$ de $50 \mathrm{~mm}$, la fuerza magneto-motriz es:

$$
f m m=3923.9 A v
$$

Si se selecciona que la bobina esté formada por 820 espiras $(\mathrm{N})$, entonces la corriente máxima es:

$$
I=\frac{f m m}{N}=\frac{3923 A v}{820}=4.7 A
$$

Finalmente, a partir de la corriente máxima, se elige construir la bobina de un alambre magneto calibre No. 17 y se cubre con Policloruro de Vinilo (PVC) para proteger y dar una mejor apariencia, como se puede observar en la Figura 4. 


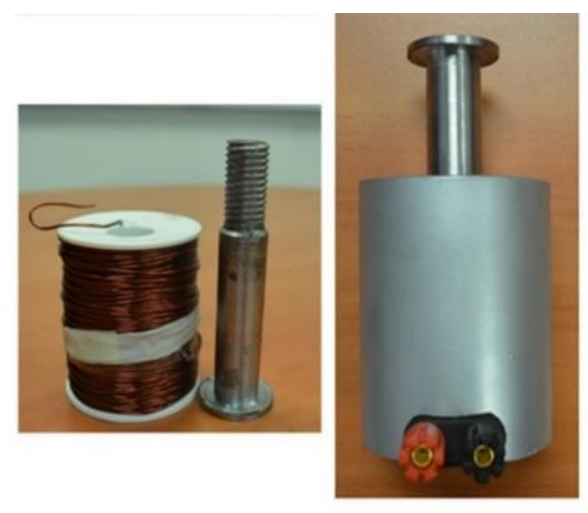

Figura 4. Electroimán del sistema.

Determinación de constante de proporcionalidad de la fuerza electromagnética $\left(C_{e}\right)$

La caracterización de la fuerza electromagnética se basa en Xie (2003) para obtener el valor de $C_{e}$. Para lo cual, se toma la siguiente condición: la posición inicial de la viga en $\theta=$ -0.0436 rad, que corresponde a los límites mecánicos del sistema. Posteriormente, se realizaron 16 mediciones de la posición de la viga y la corriente en la bobina aplicando una señal de voltaje de tipo PWM (Modulación de ancho de pulso) en los extremos del actuador magnético.

Los datos obtenidos se ajustan a una función polinomial relacionada a la corriente $i$ en función de la posición $\theta$, ver Figura 5. Obteniendo la siguiente expresión:

$$
i=812.75 \theta^{3}+50.78 \theta^{2}+2.96 \theta+0.3845
$$

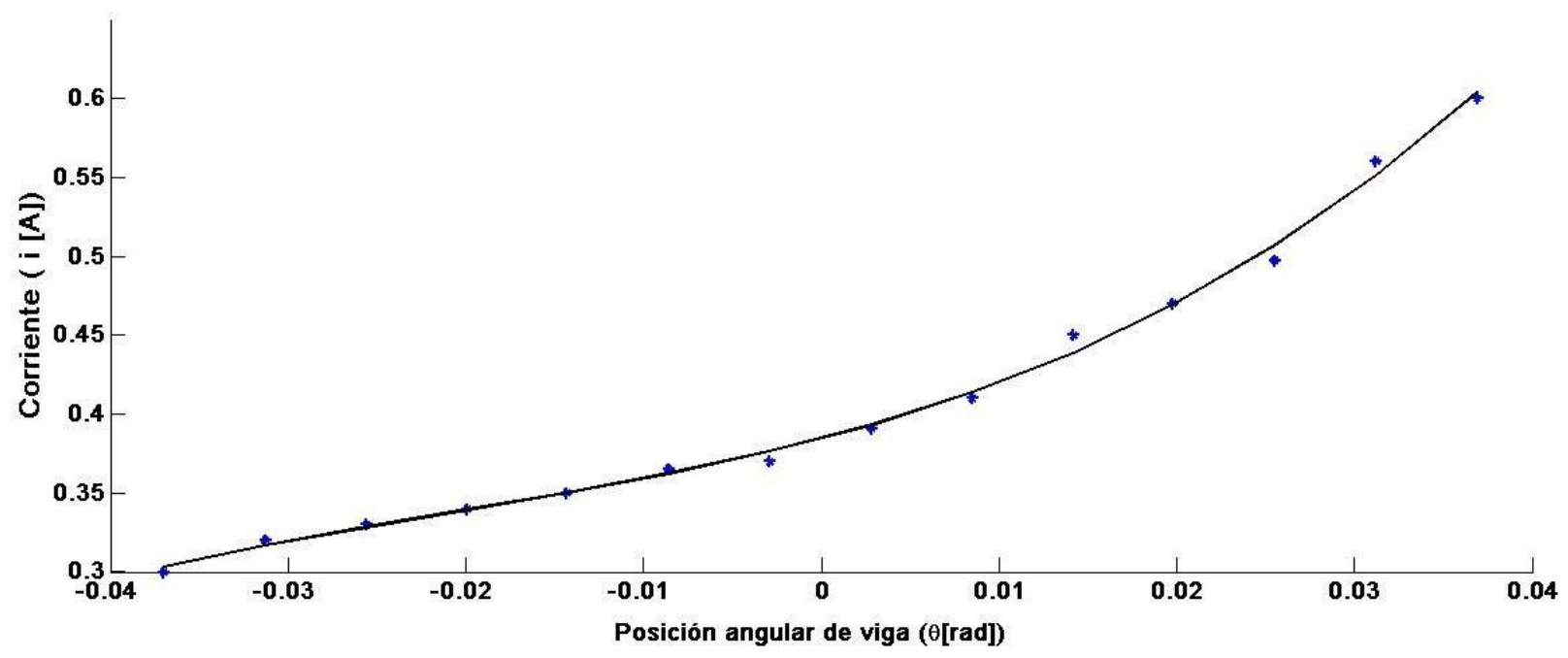

Figura 5. Comportamiento de la corriente de la bobina. 
De (1), se tiene que la constante $C_{e}$ se expresa como:

$$
C_{e}=F_{m a g} \frac{(l \sin \theta)^{2}}{i^{2}}
$$

Sustituyendo (6) y (13), y evaluando en el punto de equilibrio (0.001 $\mathrm{rad})$, se tiene el valor:

$$
C_{e}=-9.1082 \theta^{3}+0.5343 \theta^{2}+0.0006 \theta=1.1252 \times 10^{-6}\left[\mathrm{Nrad}^{2} / A^{2}\right]
$$

En la Figura 6 se muestra el comportamiento de $C_{e}$.

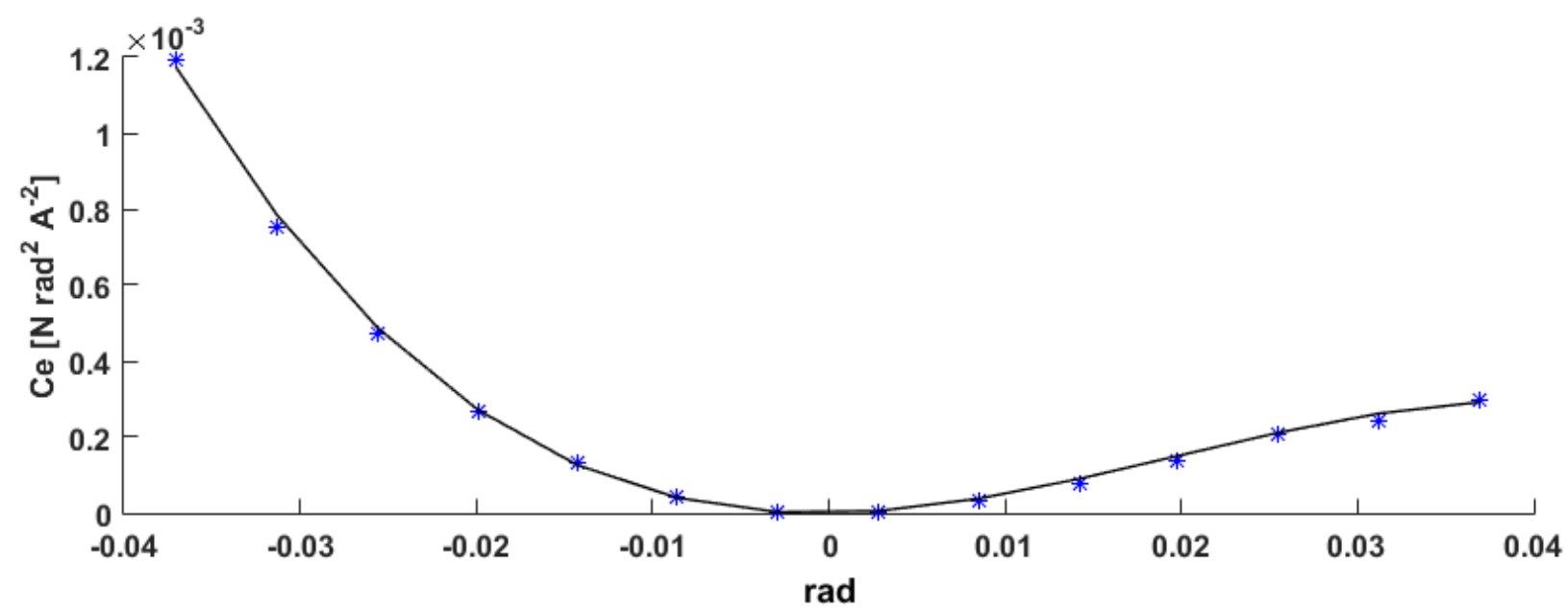

Figura 6. Comportamiento de $C_{e}$

\section{Estrategia de Control y simulación numérica}

El esquema de control propuesto para este sistema es el conocido PID. Esto, debido a que permite una implementación computacional sencilla. La función de transferencia de la ley de control se presenta en la siguiente forma (Sira, 2005):

$$
G_{c}(s)=K_{p}+K_{i} \frac{1}{s}+K_{d} s
$$

Con el fin de simplificar la expresión (5), se definen las siguientes constantes:

$$
a_{3}=2 J L l^{2}
$$




$$
\begin{aligned}
& a_{2}=2 J L l^{2} R \\
& a_{1}=L l^{2} g m r\left(1+3 R \cos 2 \bar{x}_{1}\right) \\
& a_{0}=l^{2} R g m r\left(1+3 R \cos 2 \bar{x}_{1}\right) \\
& b=4 \mathrm{C}_{e} \csc \bar{x}_{1} \sqrt{\frac{g l^{2} m r \cos \bar{x}_{1} \sin ^{2} \bar{x}_{1}}{\mathrm{C}_{e}}}
\end{aligned}
$$

Quedando:

$$
G_{p}(s)=\frac{b}{a_{3} s^{3}+a_{2} s^{2}+a_{1} s+a_{0}}
$$

Realizando el sistema en lazo cerrado de la planta con el controlador y considerando la forma simplificada de (5) y (16), se obtiene la expresión:

$$
\frac{\theta(s)}{x^{*}(s)}=\frac{\left(K_{d} s^{2}+K_{p} s+K_{i}\right) b}{a_{3} s^{4}+a_{2} s^{3}+\left(a_{1}+K_{d}\right) s+\left(a_{0}+K_{p}\right)+K_{i}}
$$

\section{Estabilidad del punto de equilibrio}

Utilizando el teorema 4.7 de (Khalil, 2002), se puede establecer localmente la estabilidad del punto de equilibrio a partir de los polos de la función de transferencia (17). En este caso, utilizando el criterio de Routh-Hurwitz (para mayores detalles ver Aguirre (2006), Aguirre (2007), Aguirre-Hernández, (2012), Aguirre-Hernández, (2017)), se tienen las siguientes condiciones:

$$
\begin{gathered}
a_{3}, a_{2,} K_{i}>0 \\
K_{p}>-a_{0} \\
K_{d}>\frac{K_{i}}{\left(a_{0}+K_{p}\right)}+\frac{a_{3}}{a_{2}}\left(a_{0}+K_{p}\right)-a_{1}
\end{gathered}
$$

Donde, las condiciones sobre $a_{3}, a_{2}$, se cumplen por la naturaleza del modelo, y el resto definen los parámetros de control que aseguran estabilidad alrededor del punto de equilibrio. 


\section{Resultados numéricos}

Con la finalidad de observar el comportamiento del sistema en lazo cerrado del sistema (17), se realizó una simulación numérica en MATLAB considerando los siguientes valores:

- Llevar la posición de la viga a $\theta=x^{*}=0.001 \mathrm{rad}$.

- Parámetros del sistema.

$m=0.595 \mathrm{~kg}$,

$l=0.2 m$,

$r=0.009 m$

$J=0.01 \mathrm{kgm}^{2}$

$R=4.5 \Omega$,

$L=0.2703 H$,

$g=9.8 \mathrm{~m} / \mathrm{s}^{2}$,

Para determinar las ganancias de las leyes de control PI y PID, se realizó la sintonización utilizando el método de Ziegler-Nichols de oscilaciones sostenidas (Sira, 2005). Se obtuvo una ganancia crítica, $k_{c}=2.5246 \mathrm{~V}$ y un periodo crítico $P_{c}=0.0609 \mathrm{~s}$. Por tanto, el controlador PID tiene los siguientes parámetros:

$$
k_{p}=1.514, k_{i}=49.72 \text { y } k_{d}=0.0115
$$

Recíprocamente, el controlador PI tiene los siguientes parámetros:

$$
k_{p}=1.136 \text { y } k_{i}=22.386
$$

En la Figura 7 se muestra la respuesta corroborando que la viga es llevada a la posición deseada con condición inicial $\theta_{0}=-0.1745 \mathrm{rad}$. 
Modelado y control de un sistema de levitación magnética basado en un cojinete magnético activo

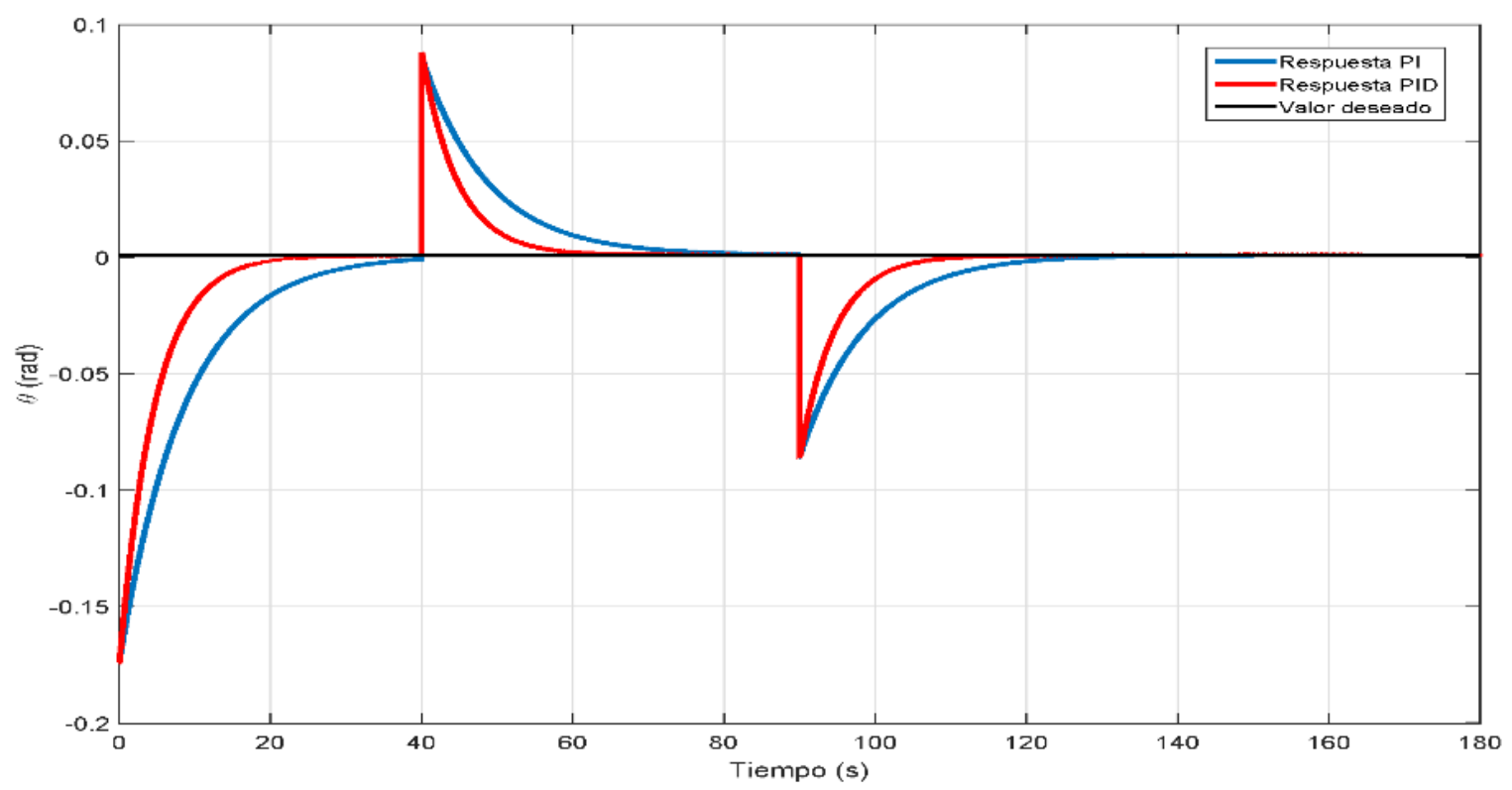

Figura 7. Comportamiento en lazo cerrado de la salida del sistema.

\section{Resultados experimentales}

En la plataforma de levitación se implementó la estrategia de control PID propuesta en la sección anterior. Los datos de la posición de la viga y la corriente del electroimán se obtienen mediante una tarjeta Discovery STM32F4 empleada como tarjeta de adquisición con un periodo de muestreo de $10 \mathrm{~ms}$. Adicionalmente, la misma tarjeta calcula y envía la señal de control al sistema modulada en PWM. En las Figuras 8 y 9, se muestran el esquema de sistema y el prototipo implementado, respectivamente.

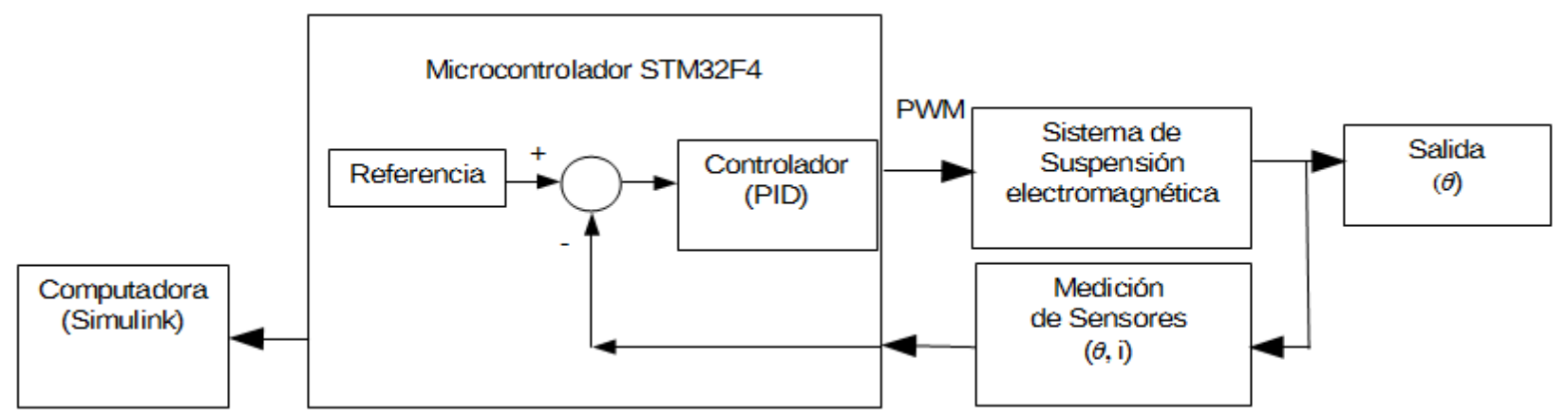

Figura 8. Esquema para la implementación. 


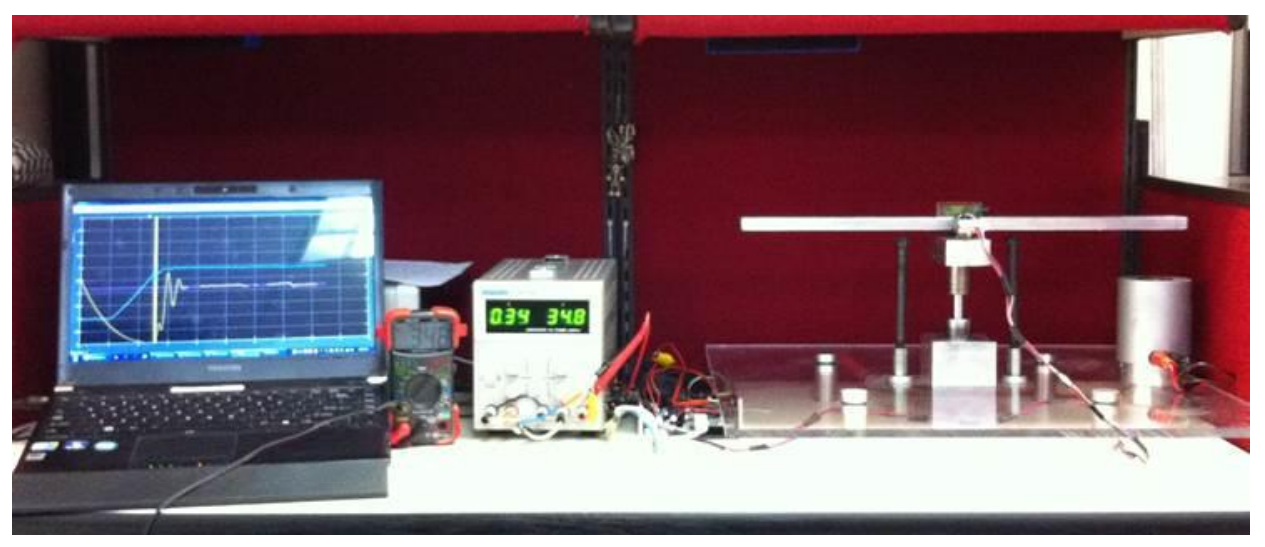

Figura 9. Plataforma experimental implementada.

El primer experimento consiste en llevar a la viga a una posición horizontal de $\theta=$ -0.0872 rad, con los parámetros del sistema y valores de ganancias del controlador descritos en (18) y (20), además de considerar la condición inicial de $\theta_{0}=-0.1745 \mathrm{rad}$ cuyo valor se encuentra por debajo de la horizontal.

En la Figura 10 se muestra la respuesta del sistema, y se observa que la salida logra la posición deseada después de 18 segundos de manera suave. La Figura 11 muestra la corriente que circula por el electroimán, y finalmente en la Figura 12 se presenta la señal de control aplicada al sistema oscilando entre $7.6 \mathrm{~V}$ y $9.3 \mathrm{~V}$.

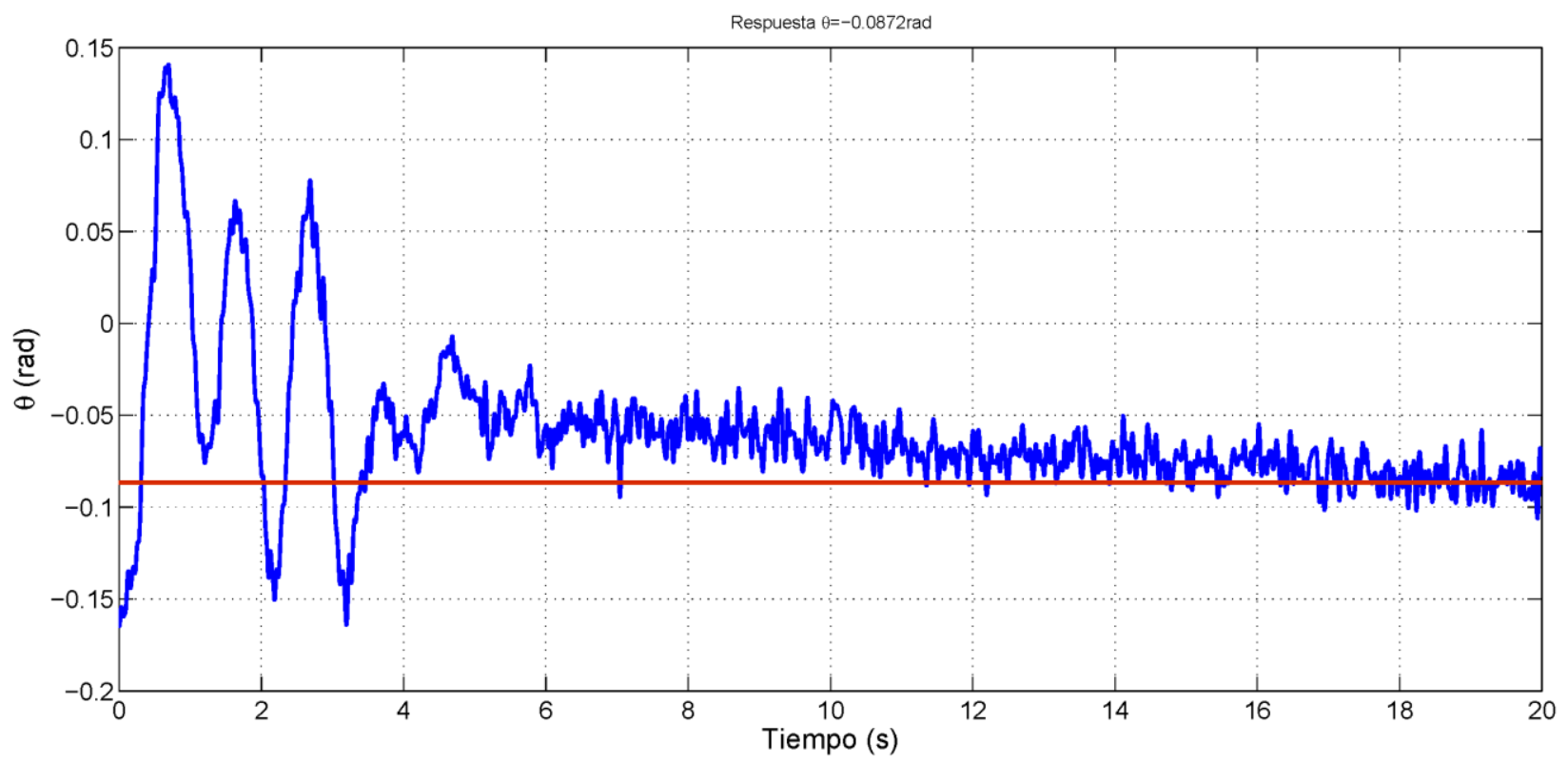

Figura 10. Respuesta de sistema. 


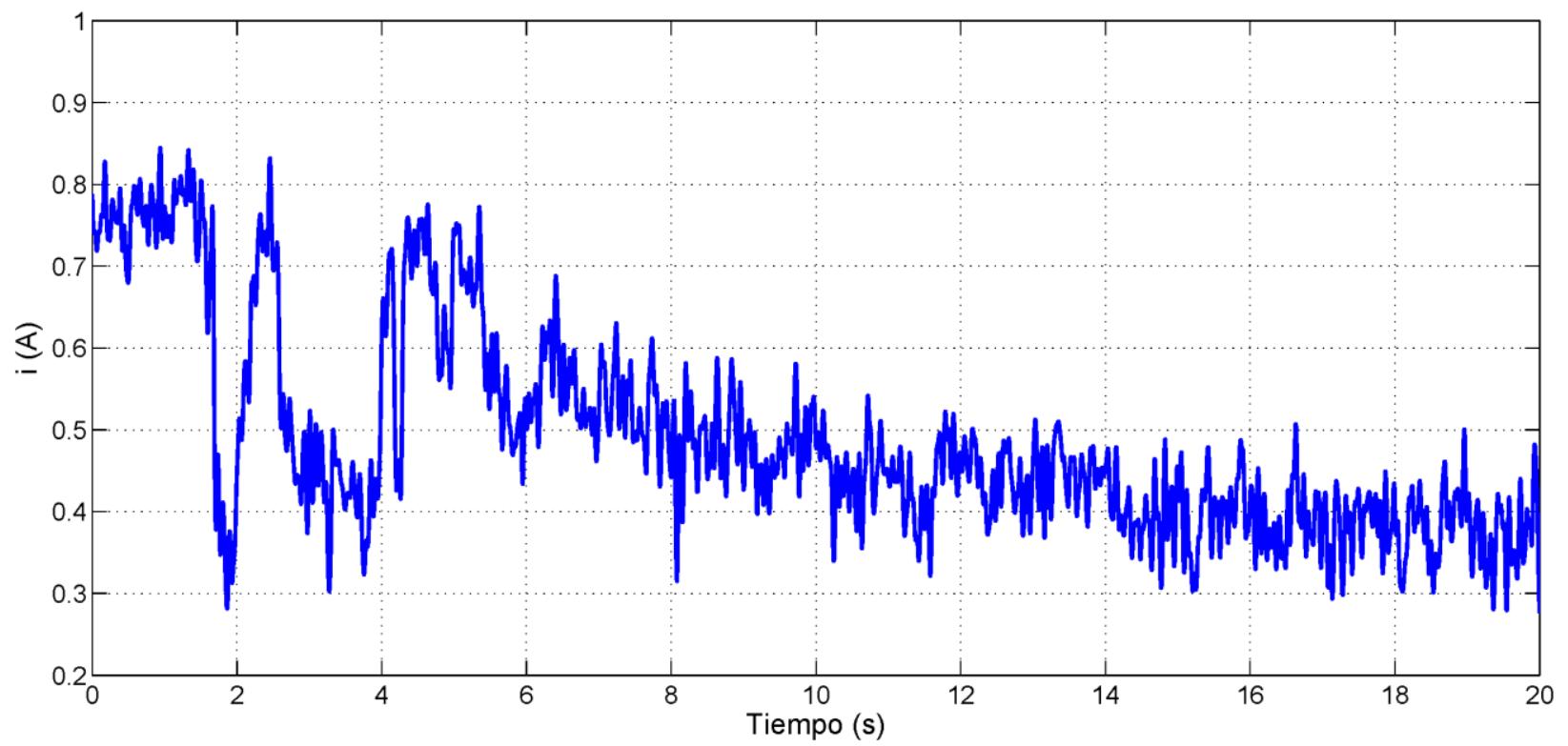

Figura 11. Corriente que circula por el electroimán.

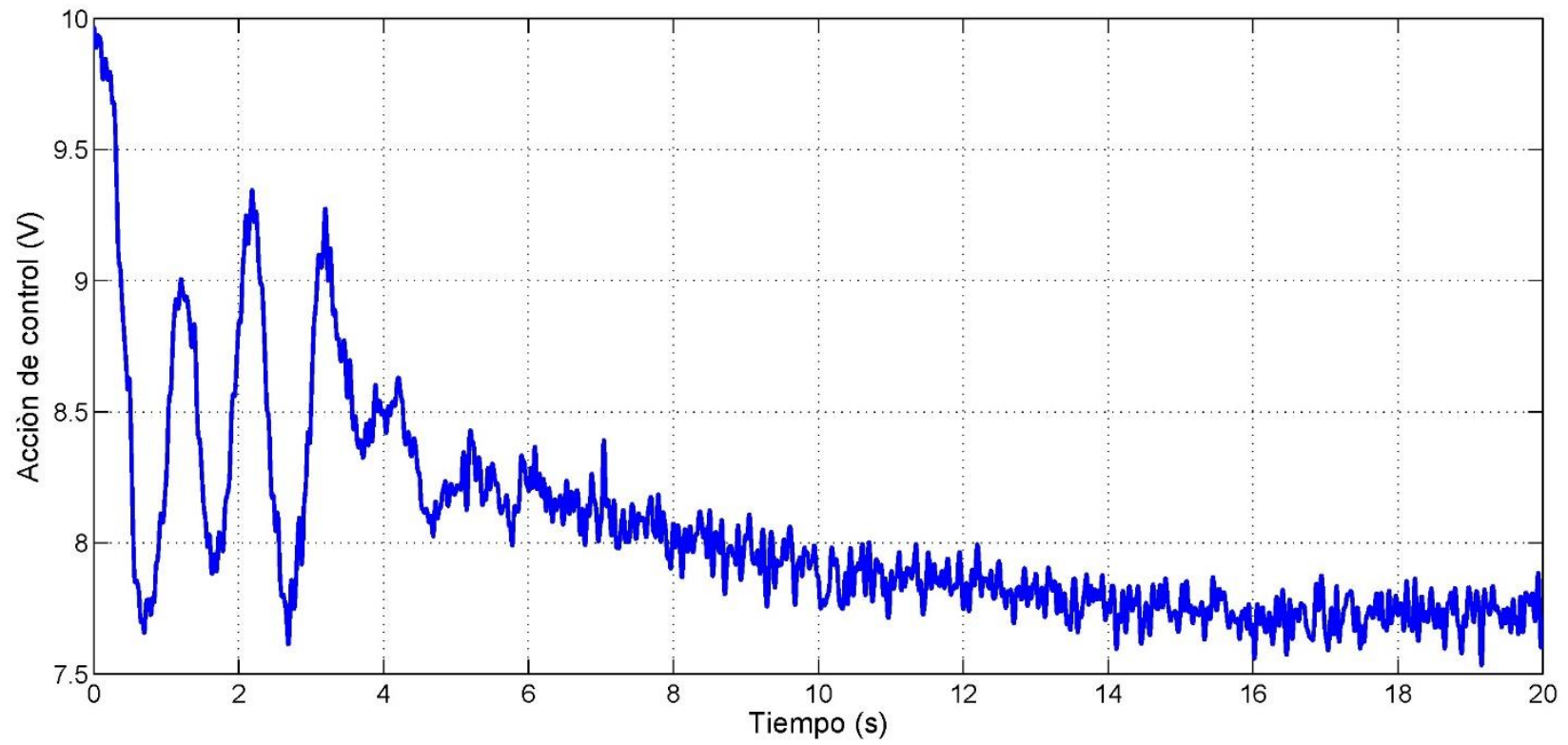

Figura 12. Señal de control aplicada al sistema.

Como segundo experimento, se colocó una carga de $0.30 \mathrm{~kg}$ con las mismas condiciones del controlador del experimento anterior, con la finalidad de generar una perturbación externa al sistema. Con ello, se busca que el sistema regrese al equilibrio a pesar de dicha perturbación; es 
decir, analizar el comportamiento de la ley de control ante perturbaciones externas. En la Figura 13 se muestra el sistema con la carga.

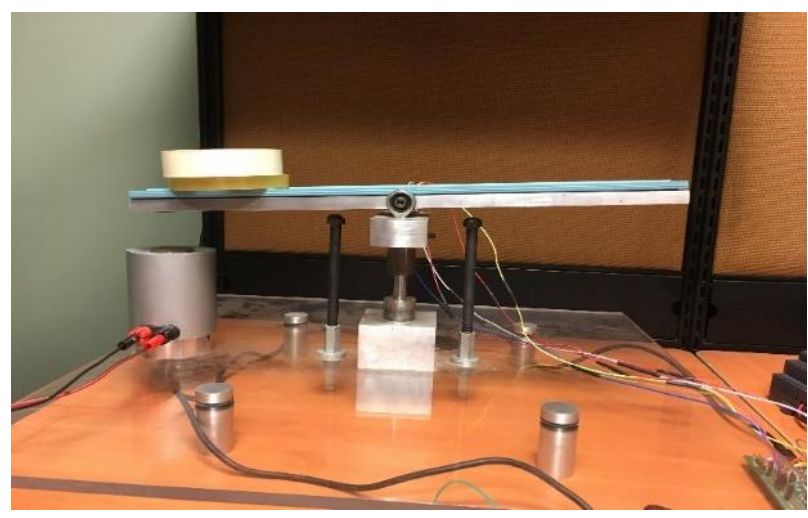

Figura 13. Sistema en presencia de carga.

En la Figura 14 se observa el comportamiento del sistema incluyendo la carga. Como primer paso se lleva la viga a la posición deseada de $\theta=0.001 \mathrm{rad}$ con una condición inicial de $\theta_{0}=$ -0.1745rad y una vez alcanzada, se coloca la carga alrededor del segundo 40 para que después de 15 segundos el sistema regrese a la posición deseada. Posteriormente, se retira la carga a los 80 segundos, recuperando el equilibrio a los 100 segundos, alcanzado la posición deseada en equilibrio. Las Figuras 15 y 16 muestran el valor de la corriente que circula por la bobina y la señal de control del sistema. La Figura 17 muestra el error de posición. 
Modelado y control de un sistema de levitación magnética basado en un cojinete magnético activo

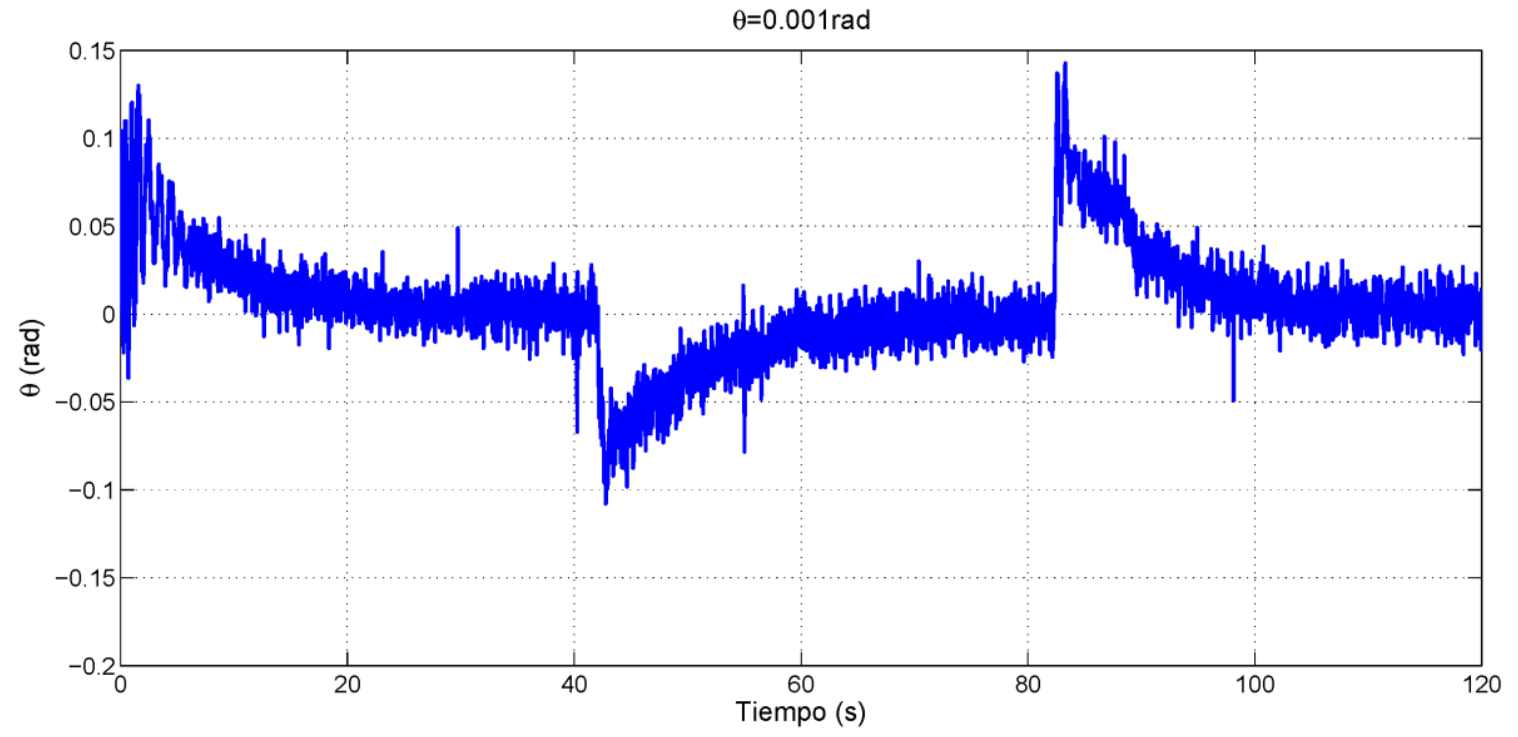

Figura 14. Respuesta de sistema con carga.

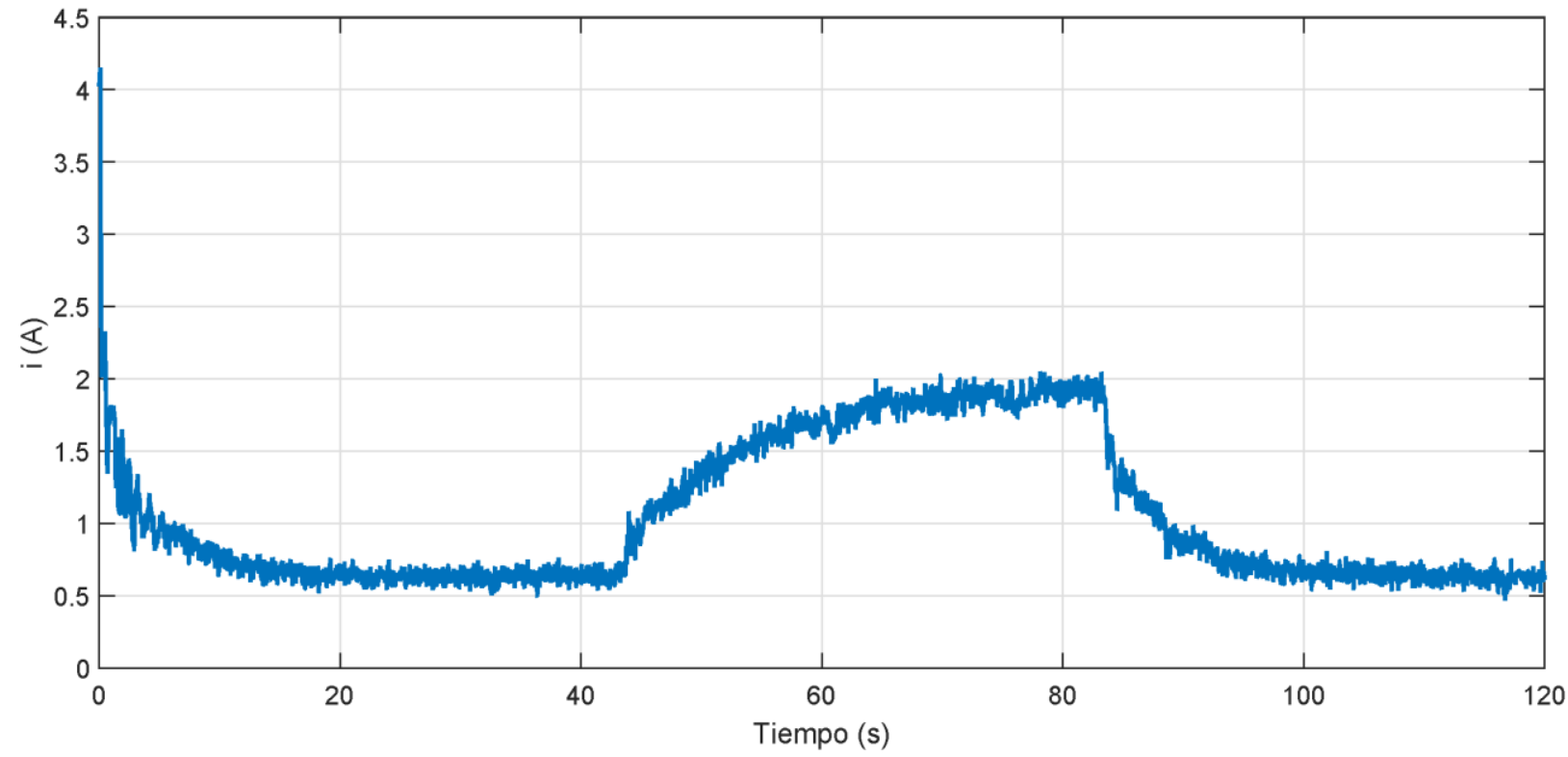

Figura 15. Corriente que circula por el electroimán. 
Cruz Pegueros, Marlon et al.

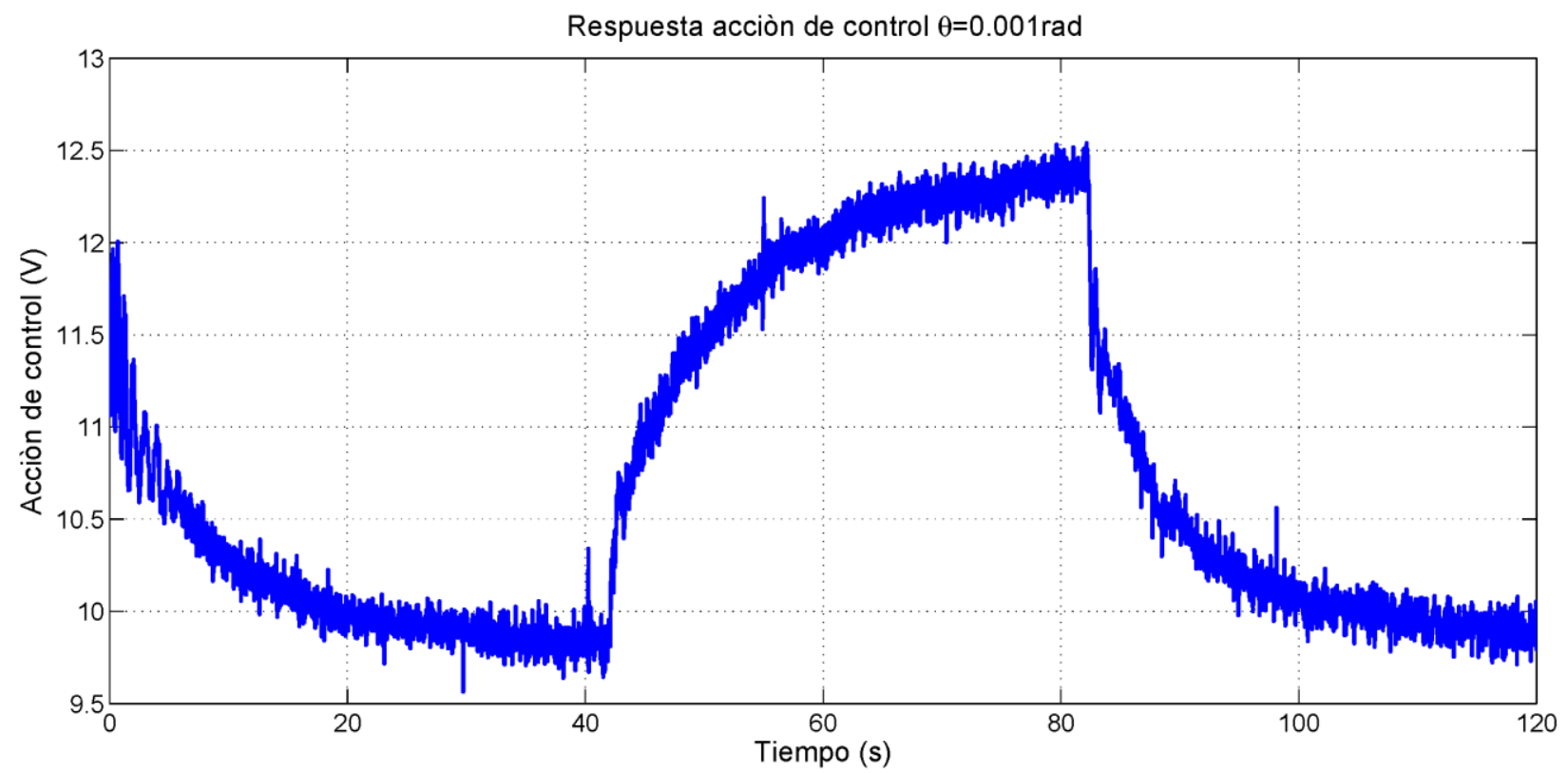

Figura 16. Señal de control aplicada al sistema.

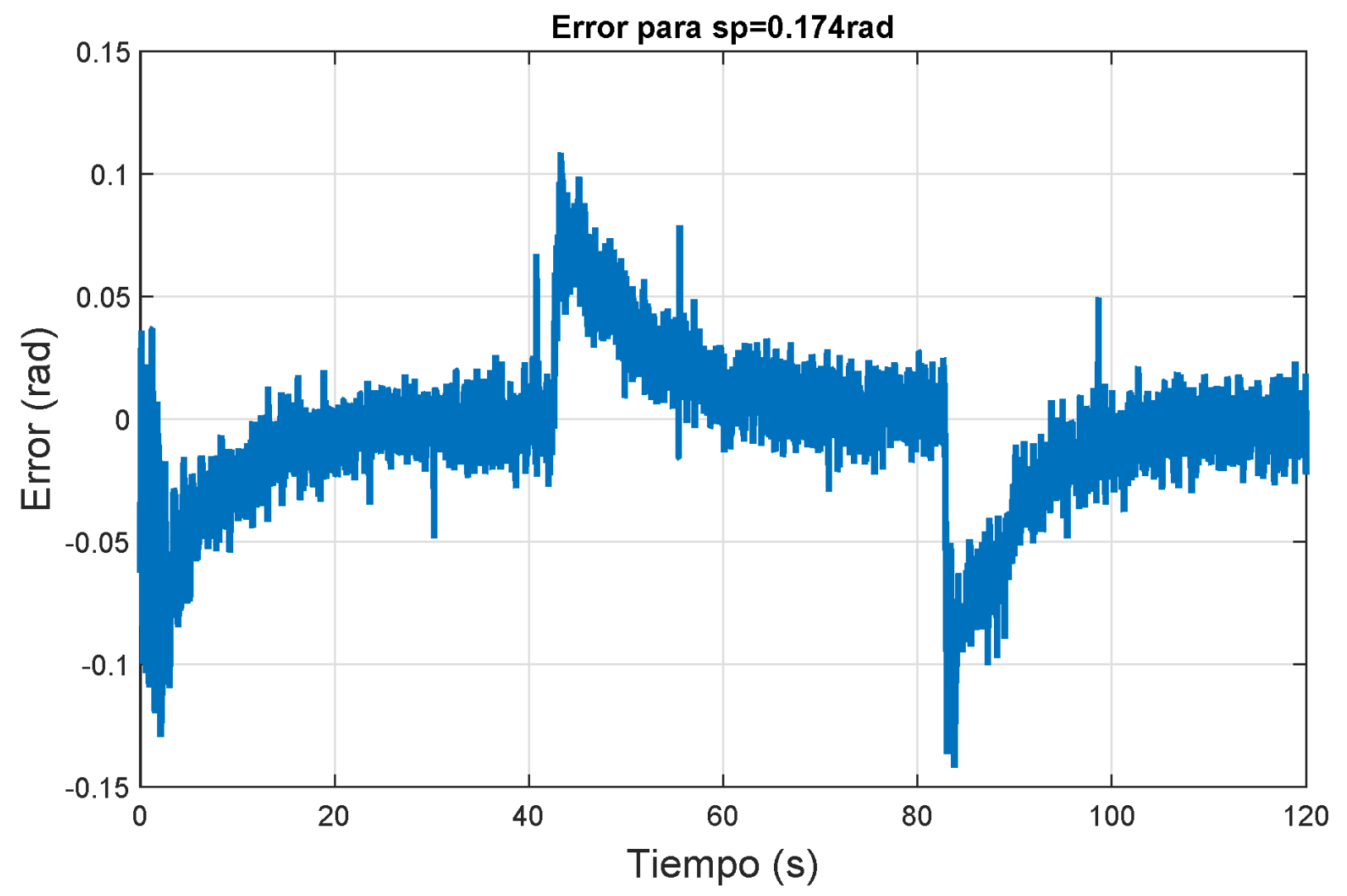

Figura 17. Error de posición. 
Para comparar el comportamiento de sistema con otro tipo de esquema de control, se implementó un controlador tipo PI, considerando que los parámetros de controlador son $\quad K_{P}=0.0345$ y $K_{i}=0.7$ y la condición inicial de $\theta_{0}=-0.1745 \mathrm{rad}$, con el fin de estabilizarlo en la posición $\theta=0.001 \mathrm{rad}$, tomando en cuenta la presencia de perturbaciones externas. Las Figuras 18, 19, 20 y 21 muestran la respuesta de la salida del sistema, la corriente que circula por el electroimán, la señal de control y el error de posición, respectivamente. De las Figuras 17 y 21 se puede ver que ambos controladores tienen errores de estado estacionario $\left(e_{s s}\right)$ con valores aproximados de $0.01 \mathrm{rad}$ para el PID y de $0.005 \mathrm{rad}$ para el PI, ambos dentro de un rango aceptable para la aplicación de manipulación de objetos.

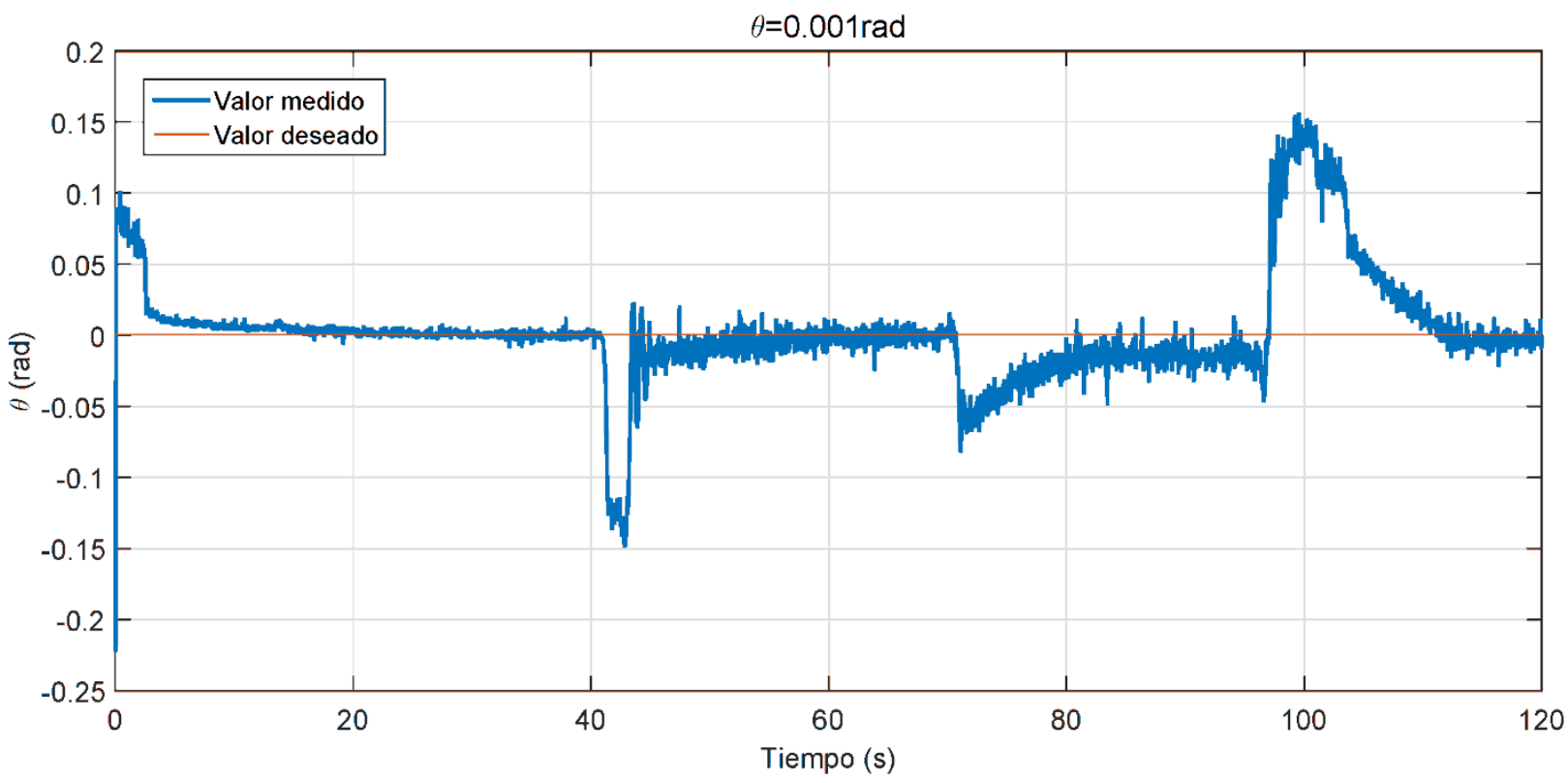

Figura 18. Respuesta de sistema con carga. 


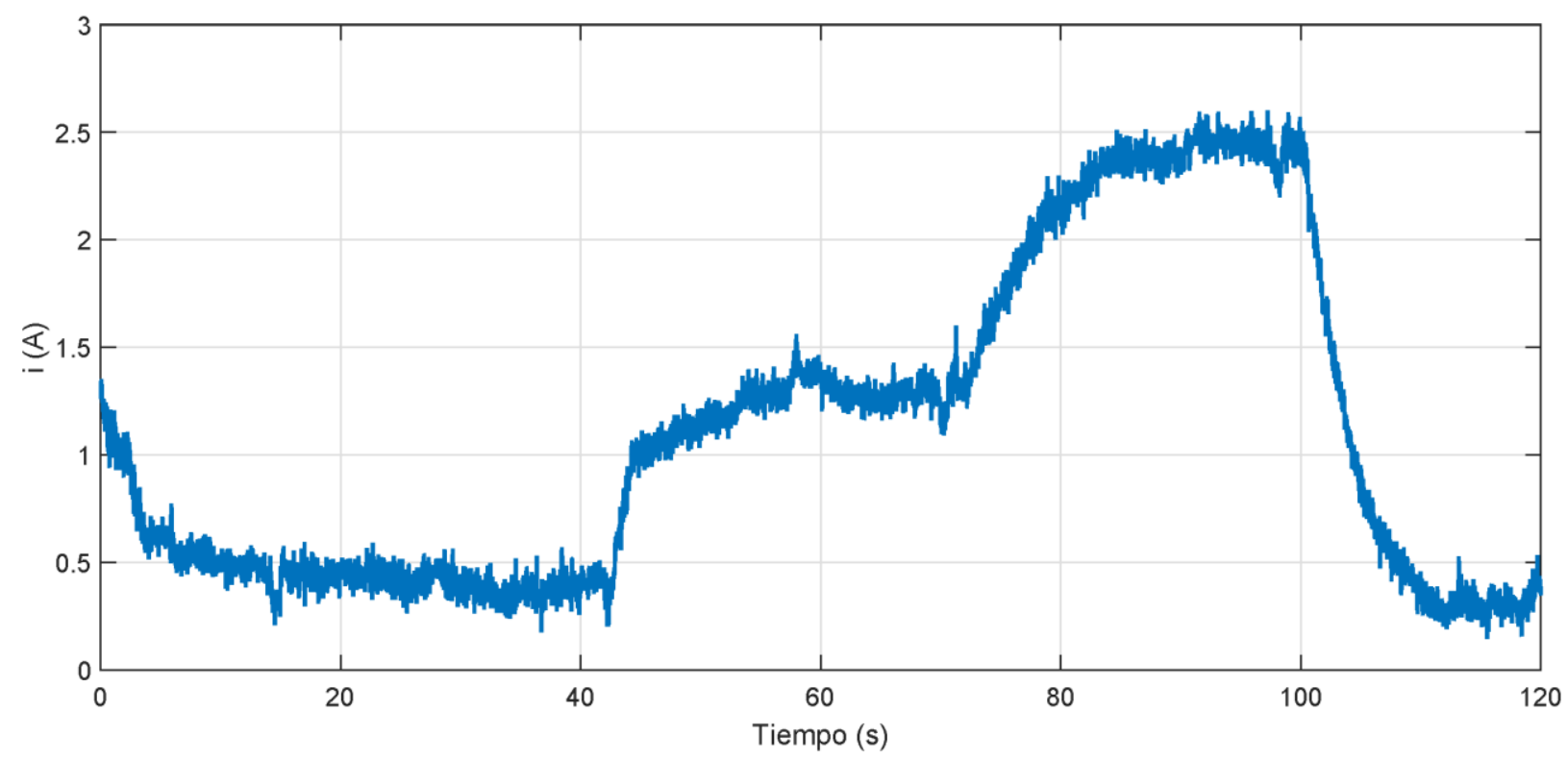

Figura 19. Corriente que circula por la bobina.

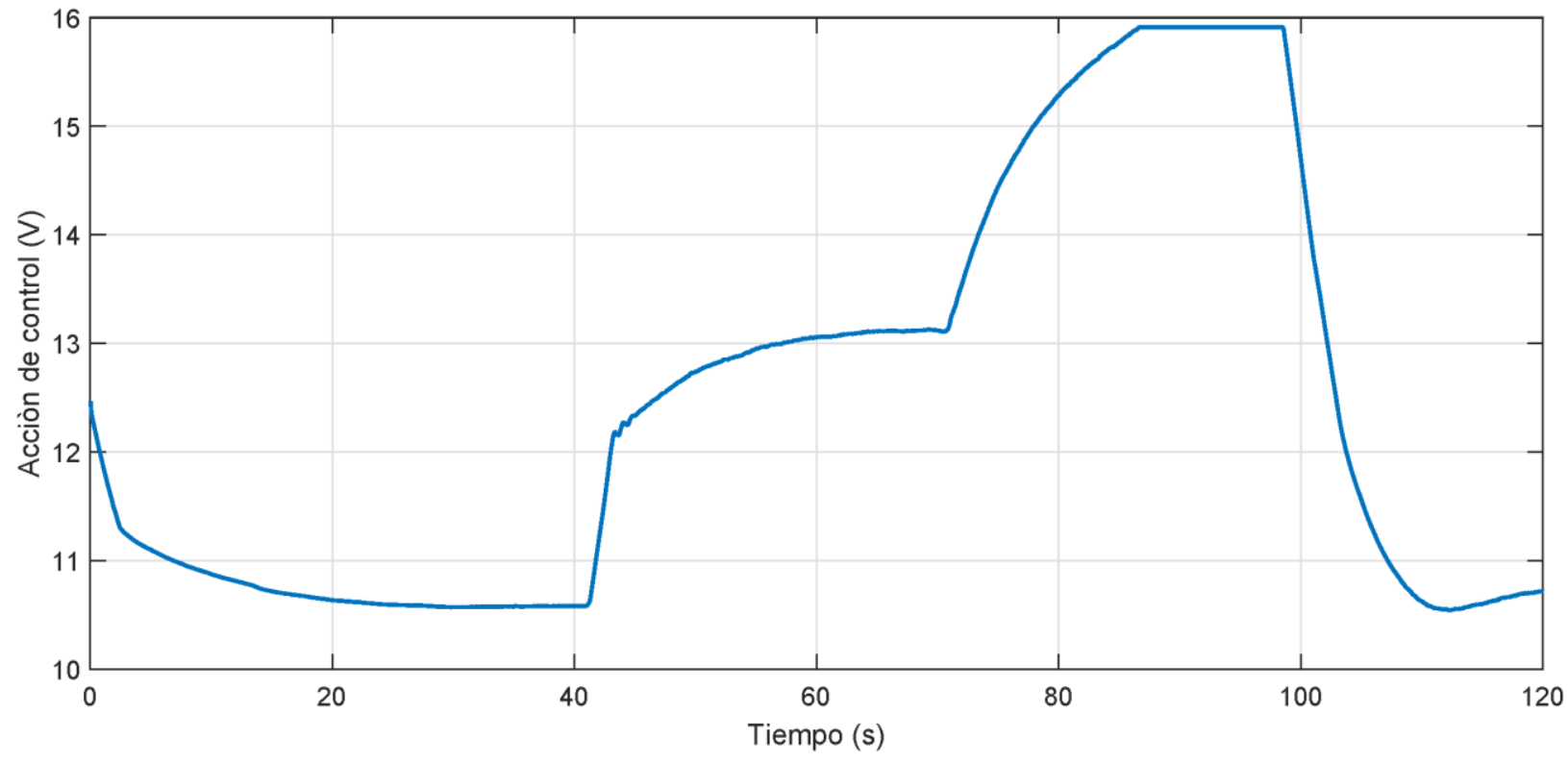

Figura 20. Señal de control aplicada al sistema. 
Modelado y control de un sistema de levitación magnética basado en un cojinete magnético activo

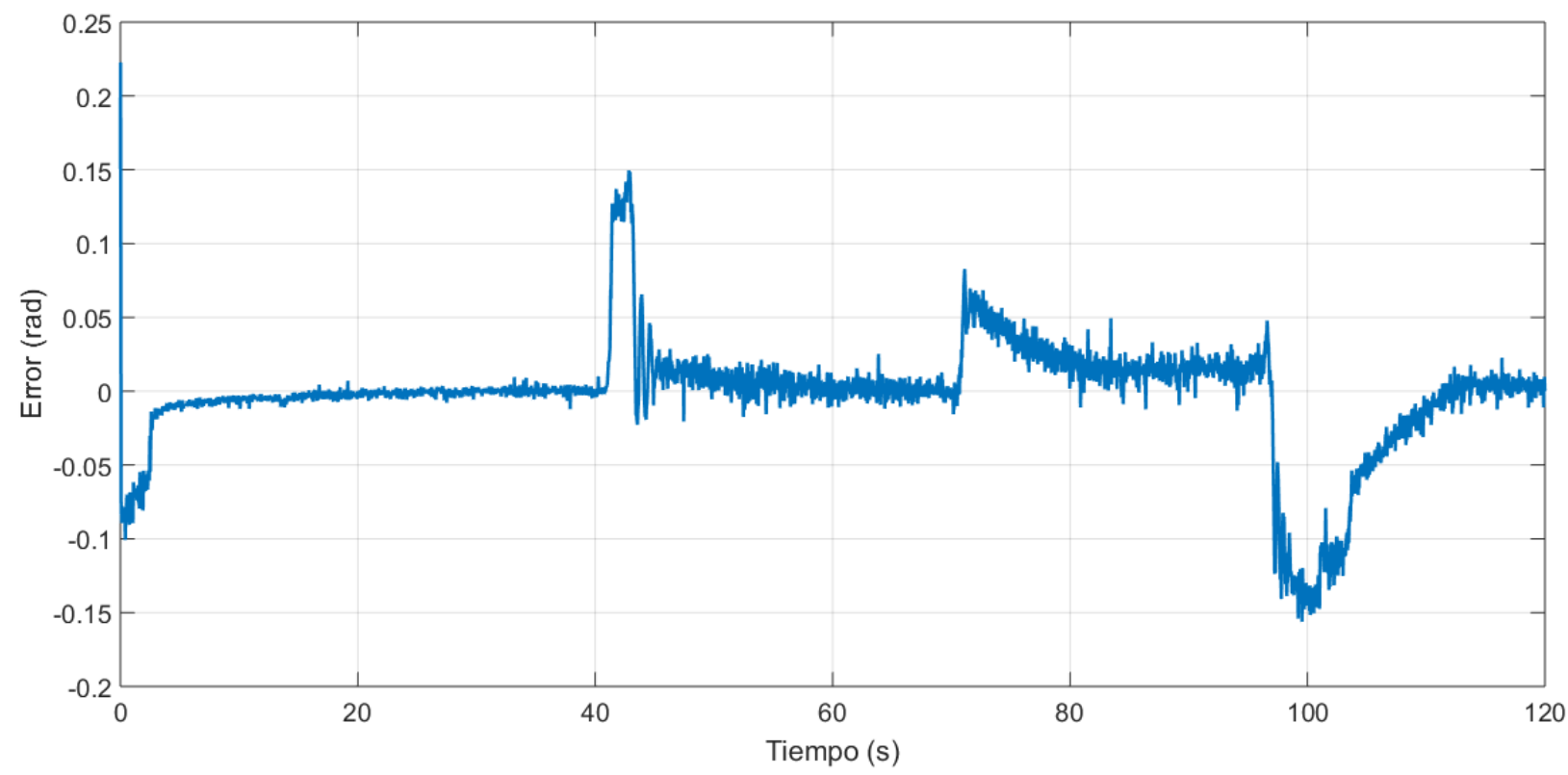

Figura 21. Error de posición.

\section{Conclusiones y trabajo a futuro}

En este trabajo se presenta el modelado y control de un sistema de levitación magnética de un grado de libertad, así como la construcción de una plataforma experimental que cuenta con la instrumentación para obtener las variables del sistema y poder implementar acciones de control.

Además, se muestra el diseño de un electroimán que tiene la función de actuador. Por otra parte, se realiza la simulación computacional del comportamiento del sistema, así como la implementación de controladores tipo PID y PI permitiendo que se realice una estabilización adecuada en la posición de equilibrio de la viga, verificándose bajo diferentes pruebas, incluyendo perturbación externa. Se plantea como trabajo a futuro el diseñar e implementar estrategias de control y estimación de estado para este sistema, considerando la dinámica no lineal, así como las saturaciones naturales de la entrada de control.

\section{Agradecimientos}

Este trabajo es financiado por la SIP-IPN, con número de registro 20170487 y 20172245. 


\section{Referencias}

Aguirre, B., \& Suárez, R. (2006). Algebraic test for the Hurwitz stability of a given segment of polynomials. Boletín de la Sociedad Matemática Mexicana, Serie 3, Vol. 12, No. 2, 263 277.

Aguirre, B.; Solis-Daun, J. and Suárez, R. (2007). Stabilization of Linear Systems: A Polynomial Approach. Advances in Dynamics, Instrumentation and Control, Vol. II, p. 21-28. Editors: Lozano, A.; Rakheja, S.; Su, Ch., World Scientific Publishing Co. Pte. Ltd. ISBN: 9812708057, 978-981270805-2.

Aguirre-Hernández, B.; Cisneros-Molina, J. L., \& Frías-Armenta, M. E. (2012). Polynomials in Control Theory Parametrized by their roots. International Journal of Mathematics and Mathematical Sciences. Volume 2012, Article ID 595076, 19 pages, ISSN: 1687-0425. DOI: $10.1155 / 2012 / 595076$.

Aguirre-Hernández, B.; Loredo-Villalobos, C. A.; Díaz-González, E.C., \& Campos-Cantón, E. (2017). Estabilidad de sistemas por medio de polinomios Hurwitz. Revista de Matemática: Teoría y Aplicaciones, 24 (1), 61-77.

Arredondo, I., Jugo, J., \& Etxebarria, V. (2008). Modeling and control of a flexible rotor system with AMB-based sustentation. ISA transactions, 47(1), 101-112.

Bächle, T., Hentzelt, S., \& Graichen, K. (2013). Nonlinear model predictive control of a magnetic levitation system. Control Engineering Practice, 21(9), 1250-1258.

Beltran-Carbajal, F., Valderrabano-Gonzalez, A., Rosas-Caro, J. C., \& Favela-Contreras, A. (2015). Output feedback control of a mechanical system using magnetic levitation. ISA transactions, 57, 352-359.

Chen, S. L., \& Weng, C. C. (2010). Robust control of a voltage-controlled three-pole active magnetic bearing system. IEEE/ASME Transactions on Mechatronics, 15(3), 381-388.

Du, H., Zhang, N., Ji, J. C., \& Gao. W. (2010). Robust fuzzy control of an active magnetic bearing subject to voltage saturation. IEEE transactions on control systems technology, 18(1), 164-169.

Golob, M., \& Tovornik, B. (2003). Modeling and control of the magnetic suspension system. ISA transactions, 42(1), 89-100.

Khalil, H. K. (2002). Nonlinear systems. Prentice Hall Upper Saddle River. 
Kim, W. J., Verma, S., \& Shakir, H. (2007). Design and precision construction of novel magnetic-levitation-based multi-axis nanoscale positioning systems. Precision Engineering, 31(4), 337-350.

Kimman, M. H., Langen, H. H., \& Schmidt, R. M. (2010). A miniature milling spindle with active magnetic bearings. Mechatronics, 20(2), 224-235.

Kummer, M. P., Abbott, J. J., Kratochvil, B. E., Borer, R., Sengul, A., \& Nelson, B. J. (2010). OctoMag: An electromagnetic system for 5-DOF wireless micromanipulation. IEEE Transactions on Robotics, 26(6), 1006-1017.

Hasirci, U., Balikci, A., Zabar, Z., \& Birenbaum, L. (2011). A novel magnetic-levitation system: design, implementation, and nonlinear control. IEEE Transactions on Plasma Science, 39(1), 492-497.

Hu, T., Lin, Z., Jiang, W., \& Allaire, P. E. (2005). Constrained control design for magnetic bearing systems. Journal of dynamic systems, measurement, and control, 127(4), 601-616.

Lindlau, J. D., \& Knospe, C. R. (2002). Feedback linearization of an active magnetic bearing with voltage control. IEEE Transactions on Control Systems Technology, 10(1), 21-31.

Lee, J. H., Allaire, P. E., Tao, G., Decker, J. A., \& Zhang, X. (2003). Experimental study of sliding mode control for a benchmark magnetic bearing system and artificial heart pump suspension. IEEE Transactions on control systems technology, 11(1), 128-138.

Lee, H. W., Kim, K. C., \& Lee, J. (2006). Review of maglev train technologies. IEEE transactions on magnetics, 42(7), 1917-1925.

Lei, S., \& Palazzolo, A. (2008). Control of flexible rotor systems with active magnetic bearings. Journal of Sound and Vibration, 314(1), 19-38.

Mahindrakar, A. D., \& Sankaranarayanan, V. (2008). State-constrained stabilization of beambalance systems. International Journal of Robust and Nonlinear Control, 18(3), 333-350.

Mahindrakar, A., \& Sankaranarayanan, V. (2007). Nonlinear control of a beam-balance system with a single electromagnet. In Proceedings of the International Conference on Advances in Control and Optimization of Dynamical Systems ACODS (pp. 340-343).

Peijnenburg, A. T. A., Vermeulen, J. P. M., \& Van Eijk, J. (2006). Magnetic levitation systems compared to conventional bearing systems. Microelectronic engineering, 83(4), 13721375 . 
Qin, Y., Peng, H., Ruan, W., Wu, J., \& Gao, J. (2014). A modeling and control approach to magnetic levitation system based on state-dependent ARX model. Journal of Process Control, 24(1), 93-112.

Rajeshkanna, G. (2012). Design, development and testing of an electromagnet for industrial waste metal scraps cleaning. International Journal of Electrical Engineering \& Technology (IJEET), 3(3), 1-7.

Rodriguez, H., Ortega, R., \& Mareels, I. (2000). A novel passivity-based controller for an active magnetic bearing benchmark experiment. In American Control Conference, 2000. Proceedings of the 2000 (Vol. 3, pp. 2144-2148). IEEE.

Sira-Ramírez, H., Marquez, R., Rivas-Echeverria, F., \& Llanes-Santiago, O. (2005). Control de Sistemas no lineales. Perason Education.

Tsuda, M., Tamashiro, K., Sasaki, S., Yagai, T., Hamajima, T., Yamada, T., \& Yasui, K. (2009). Vibration transmission characteristics against vertical vibration in magnetic levitation type HTS seismic/vibration isolation device. IEEE Transactions on Applied Superconductivity, 19(3), 2249-2252.

Wai, R. J., Lee, J. D., \& Chuang, K. L. (2011). Real-time PID control strategy for maglev transportation system via particle swarm optimization. IEEE Transactions on Industrial Electronics, 58(2), 629-646.

Xie, Y. (2003). Mechatronics Examples for Teaching Modeling, Dynamics, and Control, Doctoral dissertation, Massachusetts Institute of Technology. 